\title{
Social Interaction in a Cooperative Brain-Computer Interface Game
}

\author{
Michel Obbink, Hayrettin Gürkök, Danny Plass-Oude Bos, Gido Hakvoort, \\ Mannes Poel, and Anton Nijholt \\ Human Media Interaction, University of Twente, Enschede, The Netherlands \\ \{mobbink, gido.hakvoort\}@gmail.com, \\ $\{$ h.gurkok,d.plass,m.poel,a.nijholt\}@cs.utwente.nl
}

\begin{abstract}
Does using a brain-computer interface (BCI) influence the social interaction between people when playing a cooperative game? By measuring the amount of speech, utterances, instrumental gestures and empathic gestures during a cooperative game where two participants had to reach a certain goal, and questioning participants about their own experience afterwards this study attempts to provide answers to this question. The results showed that social interaction changed when using a BCI compared to using a mouse. There was a higher amount of utterances and empathic gestures. This indicates that the participants reacted more to the higher difficulty of the BCI selection method. Participants also reported that they felt they cooperated better during the use of the mouse.
\end{abstract}

Keywords: brain-computer interfaces, social interaction, games, cooperation.

\section{Introduction}

A brain-computer interface (BCI) is a means of interaction between humans and computers based on neural activity in the brain. It has fascinated people as it could enable whole new ways of controlling objects such as computers or wheelchairs. Since it has come into existence BCI research has mostly focused on helping disabled people, for example by controlling a wheelchair [12] or by helping them to communicate with the outside world through a word speller application [5].

Studies are currently considering applications for healthy users as well. Possibilities are applications such as virtual environment controllers [1] and games 11. An advantage of games is that when one is integrating BCI into a game one could turn a disadvantage, the lower accuracy that is associated with BCI, into a challenge that the gamer has to master [10]. This challenge could trigger a whole new genre of games where mastering your brain waves is pivotal.

One of the current main problems in BCI research is moving BCI out of the laboratory setting into the everyday environment. For BCI to perform well in

A. Camurri, C. Costa, and G. Volpe (Eds.): INTETAIN 2011, LNICST 78, pp. 183-192, 2012.

(C) Institute for Computer Sciences, Social Informatics and Telecommunications Engineering 2012 
normal situations, it has to perform when there is background noise, for example when the user is engaged in multiple tasks or when the user is collaborating with other people. A drawback of BCI is that equipment for data acquisition, such as electroencephalographs (EEGs), is very sensitive to noise. Muscle movement of the person using the BCI equipment or electrical interference might result in artifacts in the signal. As muscle movements generate artifacts users might be less inclined to interact socially with each other for worry of decreasing BCI performance. This will have consequences for cooperative applications if social interaction between users is proved to be substantially impeded.

This study looks into the influence of BCI control on social interaction in a cooperative game setting. To cooperate with each other, users should be able to interact with each other unimpeded. To study this social interaction, an environment has been setup where a player can use either a BCI or a mouse. The task was comprised of the selection of objects. This means that a BCI could be tested against a normal point and click interface with the mouse. For the BCI selection method the classification method steady-state visually evoked potentials (SSVEPs) 14 is used. This is a method that uses a flickering stimulus to activate the part of the brain where visual information is processed. When showing a group of stimuli, the player can make a selection by looking at one of the stimuli. The different stimuli each flicker on a different frequency, in such a way the stimulus that the player focuses on can be distinguished from the others. By looking at the speech, utterances, instrumental gestures and empathic gestures that players produce while playing the game the influence of BCI on social interaction was analysed.

The second section of this paper describes how to induce and measure social interaction. The SSVEP method that is used during the experiment is explained as well. The third section discusses the methodology and the game. The fourth section presents the results and in the fifth section these are discussed. Section six finishes with the conclusion and possible future work.

\section{Background}

\subsection{Inducing Social Interaction}

The first concern in social interaction research is to induce the interaction among users. According to Fowler et al. [6] and Clark [4] language is used as a coordination device, a way by which coordination among two or more individuals can be achieved to reach a common goal, or as Clark calls it: joint actions. According to Fowler et al. several studies have observed that humans have a tendency to cooperate and sometimes even imitate behaviour such as gestures, posture and verbal language. This suggests that while two users work together on a system towards the same goal, they will inherently interact with each other.

\subsection{Measuring Social Interaction}

Lindley et al. 7] measured the engagement and social behaviour of people playing a game together. The game was Donkey Konga, which could be played with 
a conventional controller and with special bongos that required the users to tap the bongos and clap their hands to the beat of the music. They treated a pair of participants as a single unit, as they did not see an individual independent from its partner. They used definitions from the Autism Diagnostic Observation Schedule (ADOS) 9] to code verbal and non-verbal behaviours. Verbal behaviour was either categorized as speech or utterances. They repeated the procedure for non-verbal behaviour, categorizing them between instrumental gestures and empathic gestures. Instrumental gestures are actions that convey a clear meaning, or are used to draw/direct attention. Gestures that could be in this category are: pointing, shrugging, nodding and moving head towards the other person. Empathic gestures are actions that convey emotion, such as placing hands in front of the mouth in shock or resting their chin on a hand. With the bongos the participants produced significantly more utterances, instrumental and empathic gestures. They showed that an alternative game controller such as the Bongos, makes participants produce more social interaction. This research is highly comparable to the current study and therefore comparable measurement methods were used. With the four categories of verbal and non-verbal behaviour all possible events were captured and by looking at the time for speech it provides a method of measuring social interaction.

\subsection{Steady-State Visually Evoked Potentials}

The SSVEP response is triggered when an user focusses on a stimulus that is flickering at a certain frequency. The SSVEP response is mostly visible between $6 \mathrm{~Hz}$ to $18 \mathrm{~Hz}$ and is recorded from the occipital region of the scalp [14. Because the power of an SSVEP response shows only over a very narrow bandwidth that corresponds to the frequency of the stimulus [8], it is detectable with a fastFourier transform (FFT). SSVEP is an exogenous event-related potential (ERP), which means that it is an involuntary brain response to an external stimulus and these occur due to internal processing of external events.

An important issue that arose when building the SSVEP system was the set of frequencies that were used and how this was presented to the user. The work of Volosyak et al. [15] present a set of possible frequencies that could be used on an LCD screen. In a small pre-experiment trial performed with 7 participants every combination of their proposed frequencies were tested to select the three frequencies that were used in this study. With an average recall of $84.6 \%$ $(\sigma=11.9)$, the set of $7.5,10$ and $12 \mathrm{~Hz}$ was selected to be used.

\section{Methodology}

\subsection{Participants}

For this study 20 participants divided into 10 pairs, were tested. All participants were asked to bring a friend. If no friend was available they were teamed up with another participant. Pairs did not have to be equal in composition, because all 
the pairs performed each selection method and therefore if the composition of a pair had influence on the interaction, it had any influence on all methods and therefore it had no effect on this study. The participants participated voluntarily in this study, and signed a consent form for their participation. To motivate the pairs to do their best a small reward, a pair of cinema tickets, was promised to the pair that completed the experiment in the shortest time. The average age of the participants was 25.25( $\sigma=7.20)$ with the youngest being 18 and the oldest 54, of the 20 participants 18 were male. Each participant had a normal, or corrected to normal eyesight, used a computer every day and at least some experience with computer games. None of the participants reported a history of epilepsy.

\subsection{The Game}

The game used in this study consisted of a playground representing a meadow (Figure 1). On this playground there were a few obstacles such as fences and vegetation and a pen. The top-down view gave the participants the ability to plan around the obstacles, and communicate their plans to each other. The playground was populated with three herding dogs and several sheep depending on the task. The goal of this game was to get all the sheep into the pen in the shortest time by giving the dogs movement instructions. By setting a goal that participants had to reach, they had something to work towards together.

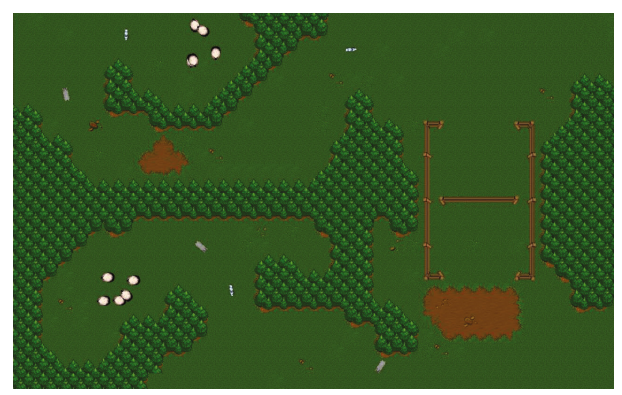

Fig. 1. A screenshot of the game containing 10 sheep and 6 dogs controlled by the players

To move the dog, the participant first moves his mouse cursor to the location the dog should move to. The participant presses and holds the left mouse button. From this moment the SSVEP method is active for the dog selection and the dogs are all highlighted with different frequencies. The participant selects the dog that has to move by looking and concentrating on the blinking stimulus of the dog that should move. As the participant holds down the mouse button the SSVEP method continues to acquires more samples over time. SSVEP detection 
has a higher accuracy over time, provided the attention of the participant is kept constant. On the other hand the participant might choose to release the mouse button sooner if a quick reaction is needed, but this decreased the chance of the correct dog being selected. So the trade-off between performance and reaction speed is up to the participant to make. If all went successfully, the correct dog moves to the location of the mouse cursor as soon as the button is released, if not a wrong dog moves to the indicated location. During the SSVEP stimulation the participant can still move the mouse cursor, altering the location the selected dog should move to.

The point and click method worked by first clicking the mouse on the dog that the participant wants to use. Once the dog is selected a small circle surrounds it as an indication of the selection. Now the participant can click on the location the dog has to move to and the dog starts moving.

\subsection{Experimental Setup}

The setup consisted of five computers: two for the participants to play on, two for the BCI acquisition and one for the recording and storing of audiovisual data. The participants were seated next to each other, as can be seen in Figure 2, so non-verbal interaction such as pointing was possible while playing the game. They both looked at their own LCD screens that were placed $50 \mathrm{~cm}$ apart from each other. This gave the participants the opportunity to turn their heads and look at each other's screen. As they had some freedom of movement and could move forward or backwards in their chairs there was no fixed distance from participant to the screen. Any movement or speaking might have impaired the accuracy of the SSVEP classifier due to muscle noise which might have lead to artefacts in the data, but it enabled them to communicate more easily at will. The participants were notified in advance that this might be the case, but they had to decide for themselves if they heeded this notification or not. The BCI caps were placed at the start of the experiment and removed at the end of the experiment. A camera and microphone were pointed at the participants as can be seen in Figure 2.

Each pair started with a short training to learn the game and the two different selection methods. Once the training was finished they played two trials of the game, once with the SSVEP selection method and once with the point and click method. Each trial took until they finished the task or a time limit of 20 minutes had passed. Each trial was played on a pre-made map. However, the layout of these maps differed, because if the same map had been used for both trials the pair might have developed a strategy on the first map and deployed it again on the second map without having to discuss this. Thereby the social interaction of the latter trial may be influenced. The maps that were used for both methods therefore differed mainly on layout and obstacles. The combination of map and selection method was selected by counterbalancing each trial. During the whole procedure the experimenter stayed in the same room. 


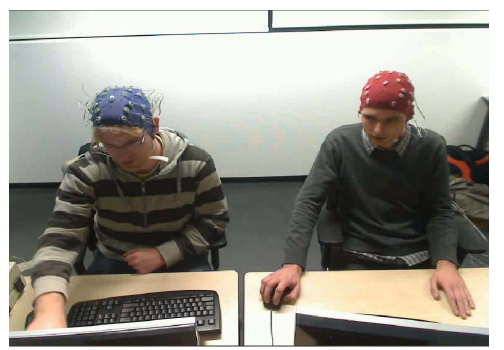

Fig. 2. One participant pointing with one hand and clenching his fist while the other participant is looking on and holding his hand flat on the tabletop

Once the experiment was completed the BCI caps were taken off and the participants were asked to fill in a questionnaire. The questionnaire asked them to think about the cooperation within the pair and rank both selection methods based on how they experienced it. It also asked them if they felt inclined to work together at all, to validate the setup of the experiment and it asked how much difficulty they had selecting a dog with each method. This might provide some correlation between difficulty and certain behaviours that were measured. Finally, the participants were interviewed about their ranking of methods in the questionnaire. By doing an interview with the participants, more information could be gathered than by asking this in the questionnaire.

\subsection{Data Acquisition, Processing and Analysis}

The SSVEP selection method used EEG signals that were acquired with a Biosemi ActiveTwo system, from five electrodes $P O 3, O 1, O_{z}, O 2$ and $P O 4$ placed according to the 10-20 international system [13. This data was digitized at $512 \mathrm{~Hz}$ sample rate, re-referenced to electrodes placed on the earlobes and analysed using Canonical Correlation Analysis (CCA) 2. CCA has advantages over the commonly used power spectral density analysis (PSDA) method introduced by Cheng et al. 3], such as a better signal-to noise ratio and no need for channel selection. CCA tries to correlate the BCI signal to a set of reference signals based on the frequencies that are used. The frequency with the highest correlation to the reference signals is selected.

The videos were annotated manually with the four behaviours that Lindley et al. 7] defined. These were speech, utterances, instrumental gestures and empathic gestures. Speech is the deliverance of formal spoken communication while utterances are all other sounds that were made by participants. Instrumental gestures are gestures that have a deliberate purpose to support cooperation, such as pointing and gazing to the others monitor. Empathic gestures are gestures that may convey the emotional state of a participant. Obvious gestures that could be thought of are gestures such as putting a hand in front of your mouth in shock, or more subtle such as increased repetitive, purposeless movement. 
Every speech and utterance component in the audio data was marked from start to finish. The total length of both speech and utterances that participants produced per trial was used for analysis. These values were normalized to a number of seconds of either per minute, because all pairs finished in different times. A pair was considered as a single unit, thus this data was averaged over the pair. The same was done with instrumental gestures and empathic gestures. These were counted after the annotation. The total number of gestures per trial for both was normalized to a number of gestures per minute for each pair. Finally all these values were averaged over all pairs and for each of the selection methods to see the differences.

In the questionnaire participants were asked to rank the selection methods based on the level of cooperation the participants experienced. In a 7-point Likert scale they were asked if they felt the need to cooperate during the experiment to measure if this study was successful in inducing interaction between participants and about the difficulty of selecting the dogs with each method.

\section{Results}

Before the results are analysed, it is important to see if this study was successful at inducing interaction between participants. An item in the questionnaire asked whether the participants felt inclined to work together. Using a 7-point Likert scale 20 subjects answered with a mode of 7 (9 out of 20 answered with a 7). Testing these results with a Wilcoxon signed-rank test to a neutral result, with an average of 4 , yielded $Z=-3.9811, p<0.001$. Therefore it can be concluded that the experiment was successful in inducing cooperation within the pairs.

Table 1. An overview of all average values, and standard deviation within parentheses, over all the pairs for each of the behaviours for both the selection methods. For speech and utterances theses values are in seconds per minute and for instrumental and empathic gestures these values are number of gestures per minute.

\begin{tabular}{|l|l|l|}
\hline & BCI selection & Point and Click \\
\hline Speech & $6.43(2.92)$ & $7.56(3.70)$ \\
Utterances & $1.78(0.63)$ & $1.18(0.51)$ \\
Instrumental gestures & $0.27(0.28)$ & $0.41(0.49)$ \\
Empathic gestures & $1.81(0.70)$ & $1.21(0.80)$ \\
\hline
\end{tabular}

In table 1 the average values over all the pairs for all the four behaviours and both selection methods are shown. There was a higher number of speech and instrumental gestures during the use of point and click selection, and a higher amount of utterances and empathic gestures during the use of BCI. BCI tasks took on average 9.64 minutes $(\sigma=5.85)$ to finish while point and click tasks took on average 8.12 minutes $(\sigma=5.07)$ in seconds to finish. This was however not a significant difference as the deviation between pairs was very high. 
Using a Wilcoxon signed-rank test $(p=0.0645)$ shows that there is a potential trend, but no significant difference between the amount of speech with BCI and point and click, but $(p=0.0059)$ on utterances, it shows that when using BCI significantly more utterances were produced compared to using point and click. There was no significant difference between BCI and point and click for instrumental gestures $(p=0.3223)$. Looking at emphatic gestures, there are clearly significantly more gestures used while playing with BCI $(p=0.0039)$ compared to point and click.

\section{Discussion}

It was expected that due to the focus that was required for selecting a dog, and the participant's knowledge that speech and movement might disturb the EEG signal during the use of BCI selection, the amount of speech and the number of instrumental gestures would be lower. As cooperation is mostly done by speech and instrumental gestures it was expected that cooperation between participants would be reduced as well. When a wrong dog is selected it causes an unexpected situation, this triggers involuntary reactions from the participants in the form of utterances and empathic gestures. The amount of utterances and the number of empathic gestures were expected to be higher.

The participants indicated in the questionnaire that they found selecting with BCI more difficult than with point and click $(Z=4.7013, p<0.001)$. However, no significant difference was found between point and click and BCI for neither speech nor instrumental gestures. For speech there was a trend towards significance.

The amount of speech and number of instrumental gestures did not change with the selection methods. In the questionnaire, participants were asked to rank how they thought they cooperated between different selection methods. They answered 17 out of 20 times that they cooperated better during the use of the point and click selection method. This was also supported by some of the participants who voiced this during the interview afterwards. They said that at times they were too busy focusing on selecting the right dog and they did not pay much attention to what the other person was doing. Further research with additional participants could reduce the effect of such an outlier that was found in the speech condition and provide proof with a significant difference.

There was a significant difference between point and click and BCI with utterances and empathic gestures. This shows that some aspects of social interaction do change with different selection methods. There were more laughs, groans, interruptions of speech and other sounds made during a BCI played game and here was a higher number of empathic gestures as well. This increase in the number of empathic gestures and amount of utterances means that more unexpected events happened that the participants reacted on. These events are mostly the selection of a wrong dog and implies the difficulty of the BCI selection. This does not mean that they produced less cooperation, but it was influenced as they had to adapt to new situations when a wrong dog was selected. 
The results from the audiovisual data indicate aspects of social interaction are affected by the higher difficulty and effort needed for BCI. The questionnaire and the interview support this, and indicate that the use of BCI noticeably influences the cooperation between participants in such a way that they cooperated better during the use of point and click.

\section{Conclusion}

This study looked at the social interaction and cooperation during a cooperative multi player game. A comparison was made between BCI selection compared to point and click selection. Measurements were taken from: audiovisual tracks, questionnaires and an interview. The audiovisual tracks were annotated marking the duration of speech and utterances, and the number of instrumental and empathic gestures. This experiment resulted in no significant difference in the amount of speech or the number of instrumental gestures, but there was a trend towards more speech when using point and click. There was a significantly higher amount of utterances and number of empathic gestures when using BCI compared to using point and click. This indicates that aspects of social interaction are affected by the use of BCI. The information provided by the questionnaire indicate this is caused by the difficulty of BCI selection and influences the cooperation in such a way that participants cooperated better during the use of point and click.

For future work it would be interesting to look deeper into the annotation and label each utterance and empathic gesture individually. This could provide more information on what kind of utterances and empathic gestures are more common during BCI. This would show for example if participants laugh or groan more during BCI.

Acknowledgements. The authors gratefully acknowledge the support of the BrainGain Smart Mix Programme of the Netherlands Ministry of Economic Affairs and the Netherlands Ministry of Education, Culture and Science. The authors would also like to thank Michiel Hakvoort for his technical support on the game and Lynn Packwood for improving the language of this paper.

\section{References}

1. Bayliss, J.: Use of the evoked potential P3 component for control in a virtual apartment. IEEE Transactions on Neural Systems and Rehabilitation Engineering 11(2), 113-116 (2003)

2. Bin, G., Gao, X., Yan, Z., Hong, B., Gao, S.: An online multi-channel SSVEP-based brain-computer interface using a canonical correlation analysis method. Journal of Neural Engineering 6(4), 46002 (2009)

3. Cheng, M., Gao, X., Gao, S., Xu, D.: Design and implementation of a braincomputer interface with high transfer rates. IEEE Transactions on Biomedical Engineering 49(10), 1181-1186 (2002)

4. Clark, H.H.: Using Language. Cambridge University Press, Cambridge (1996) 
5. Farwell, L., Donchin, E.: Talking off the top of your head: toward a mental prosthesis utilizing event-related brain potentials. Electroencephalography and Clinical Neurophysiology 70(6), 510-523 (1988)

6. Fowler, C., Richardson, M., Marsh, K., Shockley, K.: Language use, coordination, and the emergence of cooperative action. In: Coordination: Neural, Behavioral and Social Dynamics, pp. 261-279. Springer, Heidelberg (2008)

7. Lindley, S.E., Le Couteur, J., Berthouze, N.L.: Stirring up experience through movement in game play: effects on engagement and social behaviour. In: CHI 2008: Proceeding of the Twenty-Sixth Annual SIGCHI Conference on Human Factors in Computing Systems, pp. 511-514. ACM, New York (2008)

8. Lopez, M., Pelayo, F., Madrid, E., Prieto, A.: Statistical characterization of steadystate visual evoked potentials and their use in brain-computer interfaces. Neural Processing Letters 29(3), 179-187 (2009)

9. Lord, C., Risi, S., Lambrecht, L., Cook, E.H., Leventhal, B.L., DiLavore, P.C., Pickles, A., Rutter, M.: The autism diagnostic observation schedule-generic: A standard measure of social and communication deficits associated with the spectrum of autism. Journal of Autism and Developmental Disorders 30(3), 205-223 (2000)

10. Nijholt, A., Reuderink, B., Oude Bos, D.: Turning Shortcomings into Challenges: Brain-Computer Interfaces for Games. In: Nijholt, A., Reidsma, D., Hondorp, H. (eds.) INTETAIN 2009. LNICST, vol. 9, pp. 153-168. Springer, Heidelberg (2009)

11. Plass-Oude Bos, D., Reuderink, B., Laar, B., Gürkök, H., Mühl, C., Poel, M., Nijholt, A., Heylen, D.: Brain-computer interfacing and games. In: Brain-Computer Interfaces, pp. 149-178. Springer, London (2010)

12. Rebsamen, B., Burdet, E., Guan, C., Zhang, H., Teo, C.L., Zeng, Q., Ang, M., Laugier, C.: A brain-controlled wheelchair based on P300 and path guidance. In: The First IEEE/RAS-EMBS International Conference on Biomedical Robotics and Biomechatronics, pp. 1101-1106. IEEE, Piscataway (2006)

13. Reilly, E.L.: EEG recording and operation of apparatus. In: Electroencephalography: Basic Principles, Clinical Applications and Related Fields, pp. 122-142. Lippincott Williams \& Wilkins, Baltimore (1999)

14. Ruen Shan, L., Ibrahim, F., Moghavvemi, M.: Assessment of steady-state visual evoked potential for brain computer communication. In: 3rd Kuala Lumpur International Conference on Biomedical Engineering, pp. 352-354. Springer, Heidelberg (2007)

15. Volosyak, I., Cecotti, H., Gräser, A.: Impact of Frequency Selection on LCD Screens for SSVEP Based Brain-Computer Interfaces. In: Cabestany, J., Sandoval, F., Prieto, A., Corchado, J.M. (eds.) IWANN 2009, Part I. LNCS, vol. 5517, pp. 706-713. Springer, Heidelberg (2009) 